\title{
Characterisation of the wheat phospholipid fraction in the presence of nickel and/or selenium
}

\author{
Przemysław Bernat $\cdot$ Ewa Gajewska . \\ Tadeusz Bernat $\cdot$ Marzena Wielanek
}

Received: 22 April 2013/Accepted: 24 September 2013/Published online: 2 October 2013

(C) The Author(s) 2013. This article is published with open access at Springerlink.com

\begin{abstract}
The influence of nickel (Ni) and/or selenium (Se) on phospholipid composition was studied in shoots and roots of wheat seedlings. Phospholipid differences between samples were analysed using liquid chromatography/electrospray ionization-MS coupled. A total of 39 lipid species were identified. Individual phospholipids were then quantified using a multiple reaction monitoring method. In the roots, Ni toxicity was associated with an elevated level of phosphatidic acid species. In the shoots, the phosphatidylcholine/phosphatidylethanolamine ratio was about fivefold higher than in roots and decreased in Ni-treated samples. Additionally, the concentrations of phospholipid species containing $\mathrm{C}$ 18:3 fatty acid were reduced. Lipidome data were then analyzed using principal component analysis, which confirmed the compositional changes in phospholipids in response to $\mathrm{Ni}$ and $\mathrm{Ni}+\mathrm{Se}$. In contrast, the phospholipid profiles of wheat seedlings exposed to Se alone showed more similarities with the control. Together, our results suggested that the presence of Se, despite a considerable improvement of growth of Ni-treated wheat, did not counterbalance negative effect of $\mathrm{Ni}$ on the phospholipid composition in wheat roots and shoots.
\end{abstract}

Keywords Nickel · Selenium · Wheat . Phospholipids

P. Bernat $(\bowtie) \cdot$ T. Bernat

Department of Industrial Microbiology and Biotechnology, Faculty of Biology and Environmental Protection, University of Łódź, Banacha 12/16, 90-237 Łódź, Poland

e-mail: pbernat@biol.uni.lodz.pl

E. Gajewska - M. Wielanek

Department of Plant Physiology and Biochemistry, Faculty of Biology and Environmental Protection, University of Łódź, Banacha 12/16, 90-237 Łódź, Poland

\section{Introduction}

Nickel (Ni) is a common co-contaminant in soils and sediments due to anthropogenic pollution (e.g. mining, refining, metal plating) and natural emissions (van Nostrand et al. 2008). In recent years, Ni pollution has been reported from across the world, including Asia, Europe and North America (Chen et al. 2009).

Very low concentrations of $\mathrm{Ni}$ are essential for plants, however, there are reports presenting toxic effects of high concentrations of this heavy metal on plants (Chen et al. 2009). Although influence of $\mathrm{Ni}$ on plants has been studied by many researchers, the mechanisms of its toxicity are not yet fully understood. It is considered that phytotoxicity of $\mathrm{Ni}$ is associated with disturbances in different physiological and metabolic processes. In our previous papers we documented that exposure of wheat seedlings to Ni resulted in oxidative stress and changes in nitrogen and carbon metabolism (Gajewska and Skłodowska 2007, 2009; Gajewska et al. 2013). Recently we reported $\mathrm{Ni}$-induced increase in membrane permeability and alterations in the total fatty acid composition, leading to a decrease in their unsaturation, which was indicated by a reduction in the double bond index (Gajewska et al. 2012). To expand our knowledge on Ni toxicity to the cell membrane, in the present work we examined the influence of the metal on wheat phospholipids.

Selenium (Se) is not considered as an essential nutrient for plants, however at low doses has been shown to promote their growth. The beneficial effect of Se on plants is usually ascribed to its ability to enhance antioxidative capacity of cells (Xue et al. 2001). Nevertheless, at excess concentrations this metalloid is toxic to plants, which is related to the competition between Se and sulphur and subsequent production of proteins with disturbed function and structure (Hasanuzzaman et al. 2010). 
There is some evidence that Se may increase tolerance of plants to different abiotic stress factors including heavy metals (Filek et al. 2008; Hasanuzzaman et al. 2012). Therefore, the aim of our work was to check the ability of Se to alleviate Ni stress in wheat seedlings with special attention to the effect of both elements on phospholipid composition.

\section{Materials and methods}

\section{Chemicals}

1,2-Dimyristoyl-sn-glycero-3-phospho-rac-(1-glycerol) (sodium salt) [14:0/14:0 phosphatidylglycerol (PG)], 1,2-dilauroylsn-glycero-3-phosphoethanolamine [12:0/12:0 phosphatidylethanolamine (PE)], 1,2-dimyristoyl-sn-glycero-3-phosphocholine [14:0/14:0 phosphatidylcholine (PC)], L-Rphosphatidylinositol (PI) sodium salt from bovine liver, 1,2-dimyristoyl-sn-glycero-3-phospho-L-serine sodium salt [14: 0/14:0 (PS)] and 1,2-dimyristoyl-sn-glycero-3-phosphate (sodium salt) [14:0/14:0 phosphatidic acid (PA)] were purchased from Avanti Polar Lipids. The other chemicals were of HPLC grade from POCh (Poland).

Plant material and growth conditions

Wheat (Triticum aestivum L., cv. Zyta) seedlings were grown hydroponically in the diluted (1:4) Hoagland nutrient solution ( $\mathrm{pH}$ 5.8) containing $50 \mu \mathrm{M} \mathrm{Ni}\left(\mathrm{NiSO}_{4}\right)$ and $15 \mu \mathrm{M}$ Se $\left(\mathrm{Na}_{2} \mathrm{SeO}_{3}\right)$ supplied individually or simultaneously. The seedlings grown in the medium without an addition of $\mathrm{Ni}$ and Se were considered as the control. The seedlings were grown in a controlled climate room at $24{ }^{\circ} \mathrm{C}$ and $175 \mu \mathrm{mol} \mathrm{m}{ }^{-2} \mathrm{~s}^{-1}$ photosynthetic photon flux density (PPFD), with a 16-h photoperiod. After 7 days shoots and roots were harvested and growth parameters (total lengths), $\mathrm{Ni}$ and Se concentrations and the phospholipid profile were assayed.

\section{Estimation of $\mathrm{Ni}$ and Se concentrations}

For determination of $\mathrm{Ni}$ and $\mathrm{Se}$ concentrations samples of shoots and roots were wet digested in the mixture of $70 \%$ $\mathrm{HNO}_{3}$ and $30 \% \mathrm{H}_{2} \mathrm{O}_{2}(5: 1, \mathrm{v} / \mathrm{v})$ using a microwave mineralizator. Nickel concentration was determined by atomic absorption spectrometry using a Varian SpectrAA 300 spectrometer (Varian Australia Pty. Ldt., Australia) at $232 \mathrm{~nm}$. Selenium concentration was estimated by the method of Watkinson (1966) based on the measurement of fluorescence of cyclohexane-extracted selenite-2,3-diaminonaphthalene complex at $370 / 520 \mathrm{~nm}$ (excitation/emission) using a Hitachi fluorescence F-2500 spectrometer
(Hitachi Ldt., Japan). Prior to Se analysis, $\mathrm{Se}^{6+}$ present in the samples was reduced to $\mathrm{Se}^{4+}$ by boiling of the mineralizates with an addition of $6 \mathrm{M} \mathrm{HCl}$ for $1.5 \mathrm{~h}$.

\section{Lipid extraction protocol}

Lipids were extracted from three independent biological replicates of Triticum aestivum shoots and roots according to the method of Hummel et al. (2011) with modifications. Wheat shoots and roots $(\sim 0.5 \mathrm{~g})$ were crushed in a FastPrep-24 Instrument (MP Biomedicals) homogeniser with $4 \mathrm{ml}$ of the homogenous methanol:methyl-tert-butyl-ether (1:3) mixture, with $0.01 \%$ butylated hydroxytoluene (BHT) and $100 \mu \mathrm{g} \mathrm{ml}^{-1}$ PE 24:0 as internal standard. The crushed organs were stored overnight at room temperature (RT), incubated in an ultrasonication bath at RT (10 min) and centrifuged at $1,000 \mathrm{~g}$ for $10 \mathrm{~min}$ at $4{ }^{\circ} \mathrm{C}$. After adding $2 \mathrm{ml}$ of the water:methanol (3:1) mixture, the homogenate was vortexed and centrifuged at $1,000 \mathrm{~g}$ for $10 \mathrm{~min}$ at $4{ }^{\circ} \mathrm{C}$. The organic phase was transferred and evaporated. The residue was then redissolved in $1 \mathrm{ml}$ of methanol $/ \mathrm{CHCl}_{3}(4: 1, \mathrm{v} / \mathrm{v})$ and stored at $-20{ }^{\circ} \mathrm{C}$ pending analysis.

HPLC-MS separation of phospholipid classes and characterisation of phospholipid molecular species

An HP1200 series HPLC system (Agilent Technologies) was used. The LC separation was performed on a Zorbax Eclipise XDB-C18 column $(50 \mathrm{~mm} \times 4.6 \mathrm{~mm}, 1.8 \mu \mathrm{m}$ particle size; Agilent) at $35{ }^{\circ} \mathrm{C}$. The total flow rate of the mobile phases (solvent mixtures A and B) was $0.6 \mathrm{ml} \mathrm{min}{ }^{-1}$. The gradient started at $80 \% \mathrm{~B}$ and $20 \% \mathrm{~A}$, at 2 min it linearly increased to $100 \% \mathrm{~B}$, held for $14 \mathrm{~min}$, then in $2 \mathrm{~min}$ linearly decreased to $20 \% \mathrm{~B}$. The mobile phase consisted of $5 \mathrm{mM}$ ammonium formate in water (A) and $5 \mathrm{mM}$ ammonium formate in methanol (B). Prior to the use of the column, a blank gradient was run. The mass spectrometric detection was conducted on a 3200 QTRAP LC/MS/MS system from AbSciex (USA) equipped with a turbo ion spray source. MS analysis of phospholipids was performed using ESI in a negative ion mode. Nitrogen was used as curtain gas, nebulising gas, and turbo gas. The instrumental settings were as follows: spray voltage $-4,500 \mathrm{~V}$; curtain gas (CUR) 25 ; nebulizer gas (GS1) 50; turbo gas (GS2) 60; and ion source temperature $500{ }^{\circ} \mathrm{C}$. Data analysis was performed with Analyst ${ }^{\mathrm{TM}} \mathrm{v} 1.5 .1$ software (ABSciex). To perform the phospholipid species survey, an information-dependent acquisition (IDA) method, PI (precursor ion scanning) $\rightarrow$ EPI (enhanced product ion), was used. The spectra were obtained over a range from $\mathrm{m} / \mathrm{z} 100$ to 950 . The EPI scan rate was $1,000 \mathrm{amu} \mathrm{s}^{-1}$. A scan of the precursor for $\mathrm{m} / \mathrm{z} 153$ was used 
Table 1 Effect of Ni and Se treatments on the total root and shoot lengths and on Ni and Se tissue accumulation in wheat seedlings

\begin{tabular}{|c|c|c|c|c|}
\hline & Control & $\mathrm{Ni}$ & $\mathrm{Se}$ & $\mathrm{Ni}+\mathrm{Se}$ \\
\hline \multicolumn{5}{|c|}{ Total length (mm) } \\
\hline Root & $733.10 \pm 137.20$ & $370.18 \pm 113.48 * * *$ & $641.48 \pm 91.22 * *$ & $620.53 \pm 102.31^{* * * *^{\wedge \wedge \wedge}}$ \\
\hline Shoot & $176.44 \pm 13.72$ & $136.68 \pm 12.58 * * *$ & $178.57 \pm 14.03$ & $172.45 \pm 10.64^{\wedge \wedge \wedge}$ \\
\hline \multicolumn{5}{|c|}{ Ni concentration $\left(\mu \mathrm{g} \mathrm{g}^{-1} \mathrm{DW}\right)$} \\
\hline Root & $2.86 \pm 0.63$ & $1,237.55 \pm 148.36^{* * *}$ & $2.77 \pm 0.29$ & $671.99 \pm 115.01^{* * * *^{\wedge \wedge \wedge}}$ \\
\hline Shoot & $2.54 \pm 0.58$ & $81.17 \pm 5.31 * * *$ & $2.58 \pm 0.45$ & $54.89 \pm 2.12 * * *, \wedge \wedge$ \\
\hline \multicolumn{5}{|c|}{ Se concentration $\left(\mu \mathrm{g} \mathrm{g}^{-1} \mathrm{DW}\right)$} \\
\hline Root & $0.85 \pm 0.13$ & $0.90 \pm 0.23$ & $487.99 \pm 40.19 * * *$ & $495.44 \pm 25.74 * * *$ \\
\hline Shoot & $0.88 \pm 0.19$ & $0.93 \pm 0.14$ & $101.55 \pm 6.34 * * *$ & $88.89 \pm 2.81 * * *$ \\
\hline
\end{tabular}

Data are mean $\pm \mathrm{SD}$

**, *** Indicate values that differ significantly from the control at $P<0.01$ and $P<0.001$, respectively

$\wedge \wedge$ Indicate values that differ significantly from that for Ni treatment at $P<0.001$

to detect the subspecies of PA, PE, PI, PS, and scan of the precursor for $\mathrm{m} / \mathrm{z} 168$ was performed to find PC.

Based on the precursor ion analysis of head groups and fatty acyls, a comprehensive list of MRM (multiple reaction monitoring) transitions was then generated to follow fatty acyl compositions of these lipids (parent R fatty acyl fragment transitions).

The relative quantifications of PLs were performed using 14:0/14:0 $\mathrm{PE}\left(10 \mu \mathrm{g} \mathrm{ml}^{-1}\right)$ as an internal standard before the chromatographic determination step.

Data analysis

The percentage of lipids was expressed as the mean \pm SD. Significant differences between the means were assessed using the Student's $t$ test. Differences at $P<0.05$ were considered significant. Principal component analysis (PCA) was used to compare multivariate data from roots and shoots phospholipid profiles in $\mathrm{Ni}$ and/or Se presence (Markerview v1.2.1 software, ABSciex). To minimize the influence of different levels on different lipid groups, each value for a certain species of PLs was divided by the total amount of the specific group of PLs.

\section{Results and discussion}

Growth and element concentration

Exposure of wheat seedlings to $\mathrm{Ni}$ reduced the total shoot and root lengths by 23 and $50 \%$ compared to the control, respectively (Table 1 ). Treatment of wheat seedlings with Se alone did not significantly influence the shoot growth, however it led to a $12 \%$ reduction in the total root length. Compared to the seedlings subjected only to $\mathrm{Ni}$, in those treated simultaneously with $\mathrm{Ni}$ and $\mathrm{Se}$ an improvement of
Table 2 The phospholipids molecular species determined in the wheat seedlings

\begin{tabular}{llll}
\hline Molecular species & $\mathrm{m} / \mathrm{z}$ & Molecular species & $\mathrm{m} / \mathrm{z}$ \\
\hline PA C16:0/18:1 & 673 & PE C18:1/18:2 & 740 \\
PA C16:0/18:2 & 671 & PE C18:1/18:3 & 738 \\
PA C16:0/18:3 & 669 & PE C18:2/18:2 & 738 \\
PA C18:2/18:2 & 695 & PE C18:2/20:1 & 768 \\
PA C18:1/18:1 & 699 & PG C16:0/18:1 & 747 \\
PA C18:1/18:2 & 697 & PG C16:0/18:2 & 745 \\
PA C18:1/18:3 & 695 & PG C16:0/18:3 & 743 \\
PA C18:2/18:3 & 693 & PI C16:0/18:1 & 835 \\
PA C18:3/18:3 & 691 & PI C16:0/18:2 & 833 \\
PC C16:0/18:1 & 744 & PI C16:0/18:3 & 831 \\
PC C16:0/18:2 & 742 & PS C16:0/18:1 & 760 \\
PC C18:0/18:2 & 770 & PS C16:0/18:2 & 758 \\
PC C18:1/18:1 & 770 & PS C16:0/18:3 & 756 \\
PC C18:1/18:2 & 768 & PS C18:0/18:0 & 790 \\
PC C18:2/18:2 & 766 & PS C18:1/18:1 & 786 \\
PC C18:2/18:3 & 764 & PS C18:1/18:3 & 782 \\
PE C16:0/18:1 & 716 & PS C18:1/18:2 & 784 \\
PE C16:0/18:2 & 714 & PS C18:2/18:3 & 780 \\
PE C18:0/18:1 & 744 & PS C18:3/18:3 & 778 \\
PE C18:1/18:1 & 742 & & \\
\hline PA ph0sph:3 & & \\
\hline
\end{tabular}

$P A$ phosphatidic acid, $P C$ phosphatidylcholine, $P E$ phosphatidylethanolamine, $P I$ phosphatidylinositol, $P G$ phosphatidylglycerol, $P S$ phosphatidylserine, $\mathrm{m} / \mathrm{z}$ mass to charge ratio

the total shoot length by approximately $26 \%$ was observed. Application of Se to the Ni-containing medium resulted also in an increase in the total root length by $68 \%$, compared to the seedlings treated only with $\mathrm{Ni}$. In line with our results, Se-induced growth improvement has previously been reported for plants subjected to $\mathrm{Ni}$ (Hawrylak et al. 2007) and Cd (Filek et al. 2008) stress. 
Table 3 Comparison of phospholipid concentration in the wheat roots and shoots exposed to Ni and/or Se

\begin{tabular}{lllll}
\hline & Control & $\mathrm{Ni}$ & $\mathrm{Se}$ & $\mathrm{Ni}+\mathrm{Se}$ \\
\hline Total amount $\left(\mu \mathrm{g} \mathrm{g}^{-1} \mathrm{FW}\right)$ & & & & \\
Root & $53.13 \pm 3.61$ & $44.1 \pm 0.95$ & $60.47 \pm 12.86$ & $54.58 \pm 5.3$ \\
Shoot & $75.62 \pm 6.51$ & $37.85 \pm 7.3^{*}$ & $59.27 \pm 1.51$ & $41.64 \pm 4.42^{* *}$ \\
\hline
\end{tabular}

Data are mean \pm SD

*, ** Indicate values that differ significantly from the control at $P<0.05$ and $P<0.01$, respectively

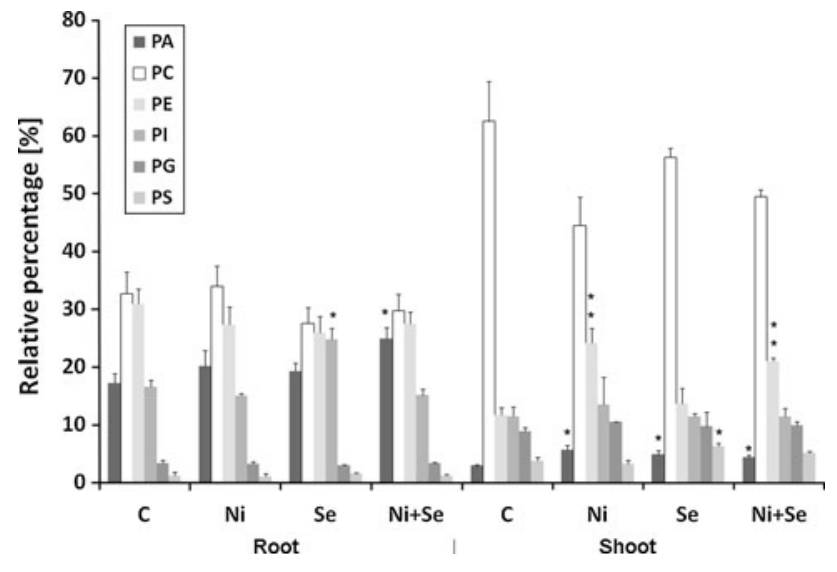

Fig. 1 Comparison of phospholipid composition of wheat roots and shoots exposed to Ni and/or Se. $P A$ phosphatidic acid, $P C$ phosphatidylcholine, $P E$ phosphatidylethanolamine, $P I$ phosphatidylinositol, $P G$ phosphatidylglycerol, $P S$ phosphatidylserine; *, ** indicate values that differ significantly from the control at $P<0.05$ and $P<0.01$, respectively

Exposure to Ni led to considerable accumulation of this metal in wheat tissues, mostly in the roots which contained about $94 \%$ of the total amount of the metal taken up by the seedlings (Table 1). Nickel stress did not influence Se uptake by the seedlings, however addition of $\mathrm{Se}$ to $\mathrm{Ni}$ containing medium significantly reduced accumulation of $\mathrm{Ni}$ both in shoots and roots, by 32 and $46 \%$, respectively, compared to the seedlings exposed only to Ni. Similarly to our results, a reduction in heavy metal uptake due to $\mathrm{Se}$ supplementation was reported by other authors (Shanker et al. 1996; He et al. 2004; Filek et al. 2008). Shanker et al. (1996) suggested that limitation of heavy metal uptake by Se might be related to the reduction of the metal availability for plants due to the formation of non toxic Se-metal complexes.

\section{Characterisation of phospholipid molecular species}

Tandem MS/MS analysis of phospholipid species in a negative ion mode reveals structural information on fatty acid chains at sn- 1 and sn-2 position of the phospholipid. Therefore, MS/MS experiments were performed to confirm the identities of two fatty acid chains of each species in all

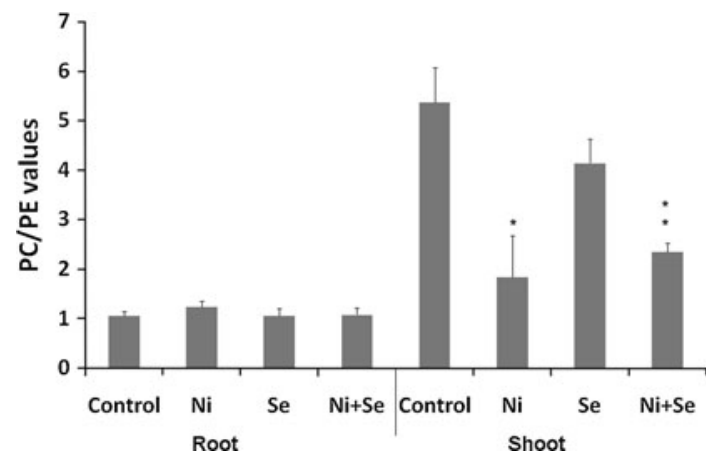

Fig. 2 The changes of PC/PE values in roots and shoots of wheat seedlings treated with $\mathrm{Ni}$ and/or Se. *, ** indicate values that differ significantly from the control at $P<0.05$ and $P<0.01$, respectively. $P C$ phosphatidylcholine, $P E$ phosphatidylethanolamine

detected phospholipid classes. Table 2 shows molecular species in each phospholipid class identified in the roots and shoots of wheat seedlings. Using the LC/ESI-MS/MS procedure, 39 species of PLs were identified, and the number of identified species for PA, PC, PE, PG, PI, PS were $9,7,8,3,3$ and 9 , respectively. Further quantitative analysis (Table 3) of the major PLs for both organs revealed that the total amount of phospholipids was the lowest in the shoots of wheat seedlings treated with $\mathrm{Ni}$ both used individually and in combination with Se. Also, significant decreases in the content of phospholipid classes were reported in cadmium stressed tomato (Ouariti et al. 1997; Ben Ammar et al. 2007).

Figure 1 shows the composition of PLs for wheat seedlings in $\mathrm{Ni}$ and/or Se presence. In the roots and shoots, PC was the predominant PL. Other determined PLs classes were PE, PI, PA, PG and PS. The same PLs classes were described by Pereyra et al. (2006) in Triticum aestivum roots. Overall, the amounts of different groups of PLs showed differences between roots and shoots. In the roots, the dominant species were PC and PE in control samples accounting for 32 and $30 \%$, respectively, whereas in the shoots the PC class was about $62 \%$. Statistically significant differences, were most often, observed in the PA class. PA constituted around 18 and $3 \%$ of total cell phospholipids in control samples of roots and shoots, respectively. When comparing control samples with those exposed to the 
Fig. 3 Relative abundances for major species of phospholipids in roots of wheat seedlings. Control (black bar), Nickel (light grey bar), Selenium (white bar) and $\mathrm{Ni}+\mathrm{Se}$ (dark grey bar). $P A$ phosphatidic acid, $P C$ phosphatidylcholine, $P E$ phosphatidylethanolamine, $P I$ phosphatidylinositol, $P G$ phosphatidylglycerol, $P S$ phosphatidylserine; *,**, *** indicate values that differ significantly from the control at $P<0.05, P<0.01$ and $P<0.001$, respectively

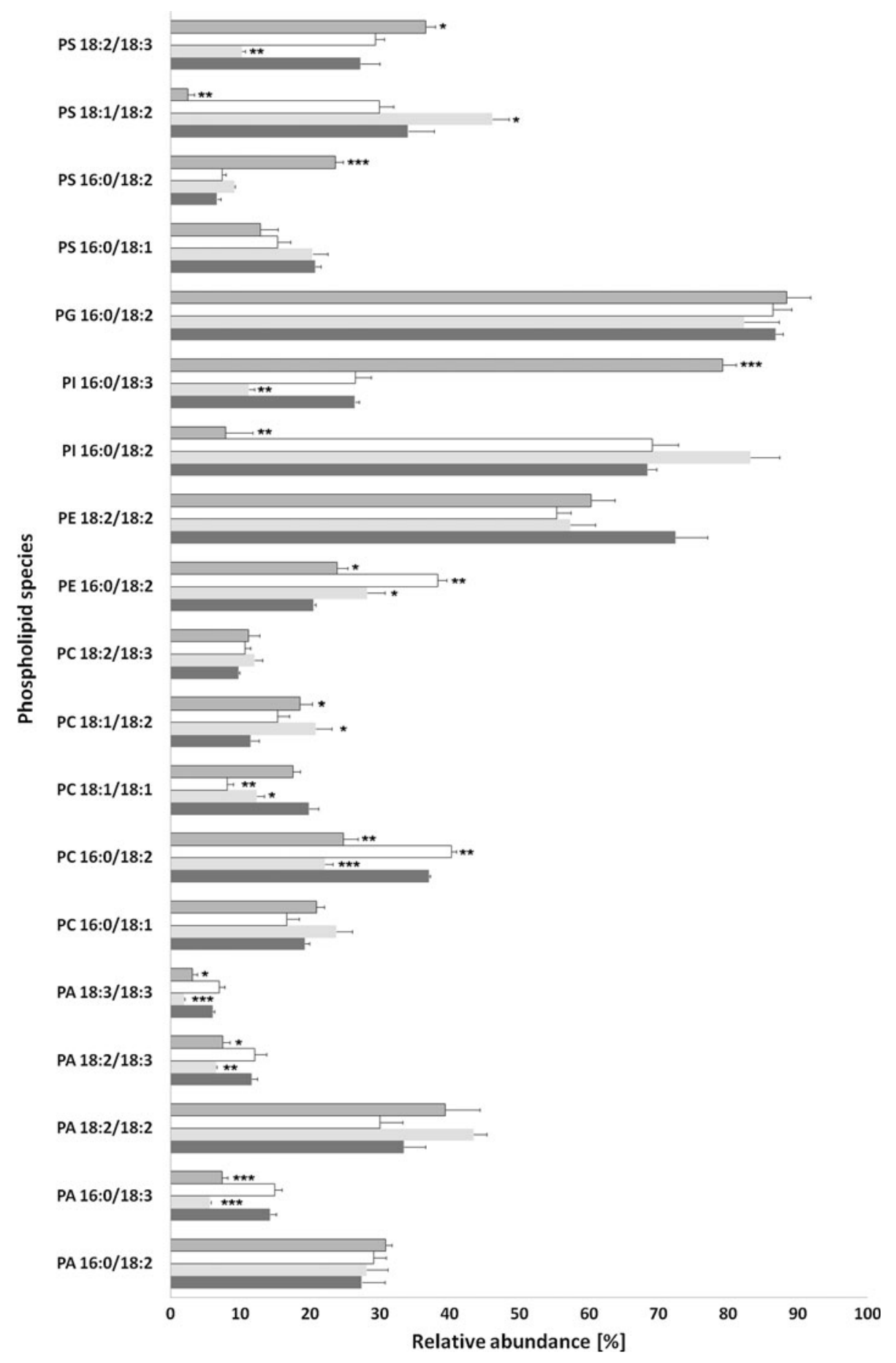

elements, relatively high amount of PA was found in the roots exposed to $\mathrm{Ni}+\mathrm{Se}$. PA level significantly increased also in $\mathrm{Ni}$ and/or Se treated shoots. Normally, PA constitutes only a minor proportion of the cellular lipid pool but, in plants, in response to different conditions of stress factors, such as bacterial and fungal pathogens, $\mathrm{H}_{2} \mathrm{O}_{2}$, chilling, salts, wounding, heavy metals exposure, PA levels can increase significantly (Testerink and Munnik 2005; Darwish et al. 2009). In the wheat shoots, significant increases in PA species were accompanied by a lower level of PC. These results suggest possible conversion of PC species to PA via a phospholipase D (PLD) pathway (Testerink and Munnik 2005).

Phosphatidylcholine and phosphatidylethanolamine are the two major membrane components of both prokaryotic and eukaryotic cells (Sutoh et al. 2010). PC forms a bilayer structure, whereas PE molecules are nonbilayer PLs (Mansour et al. 2002). In addition, a high level of choline 
Fig. 4 Relative abundances for major species of phospholipids in shoots of wheat seedlings.

Control (black bar), Nickel

(light grey bar), Selenium (white bar) and $\mathrm{Ni}+\mathrm{Se}$ (dark grey bar). $P A$ phosphatidic acid, $P C$ phosphatidylcholine, $P E$ phosphatidylethanolamine, $P I$ phosphatidylinositol, $P G$ phosphatidylglycerol, $P S$ phosphatidylserine; *,**, *** indicate values that differ significantly from the control at $P<0.05, P<0.01$ and $P<0.001$, respectively

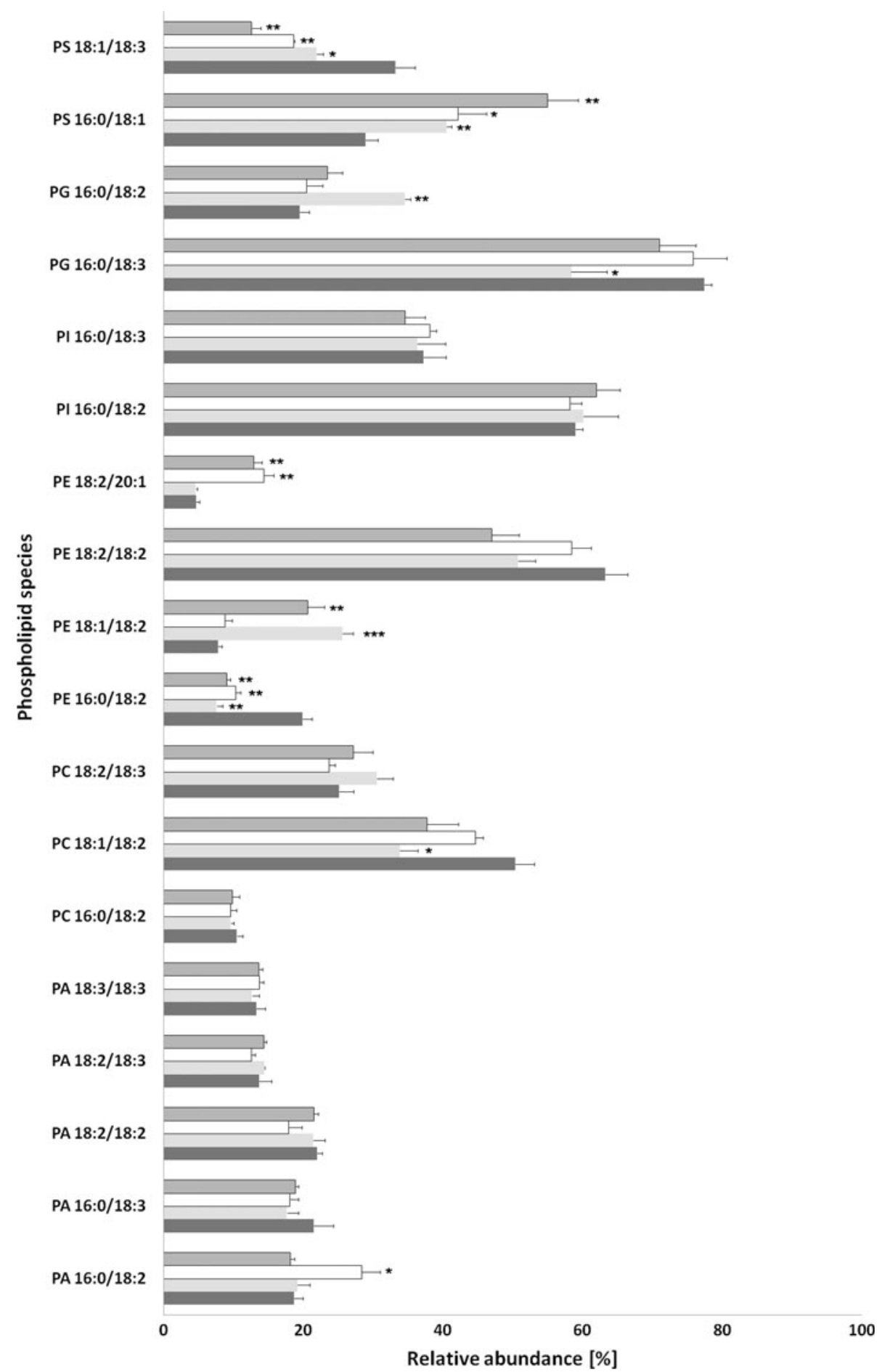

in the polar head group has a fluidizing effect on the membrane, whereas ethanolamine makes the membrane more rigid (Quartacci et al. 2001). Thus, the relative compositional change of phospholipid species in living organisms resulted in the modifications of the PC/PE ratio (Quartacci et al. 2001). In our study, the ratio in control shoots was about fivefold higher than in roots (Fig. 2).
Treatment of wheat seedlings with Ni used individually or in combination with Se induced a statistically significant increase in the PE level in the shoots (Fig. 1), which resulted in a marked decline in the PC/PE ratio. It seems that Ni presence causes lipid homeostasis disorders, possibly leading to a decrease in the membrane fluidity. A similar phenomenon was observed in wheat roots grown in 
copper excess, which may have led to enriched nonlamellar-forming domains and phase separation of nonlamellar-forming lipids (Quartacci et al. 2001).

PI contents in the control wheat seedlings reached 16 and $10 \%$ in roots and shoots, respectively (Fig. 1). However, a lower content of PI was noted in the roots of wheat Triticum durum (Quartacci et al. 2001). Among the obtained samples, statistically significant increase in PI content was observed in the roots exposed to Se.

Phosphatidylglycerol is the major phospholipid in the thylakoid membranes of plant chloroplasts (Welti et al. 2003). PG is considered to be essential for growth and development of the chloroplast (Su et al. 2009). In our study, shoots contained higher amount of PG comparing to roots. However, the content of PG did not show significant alterations in wheat seedlings in the elements presence.

Phosphatidylserine was a minor lipid species, comprising less than $6 \%$ in the shoots and trace amounts of total PLs in the roots (Fig. 1). Small amounts of PS were also described for other plants (Vance and Steenbergen 2005). Although PS is a minor phospholipid component of plant membranes, it is likely to have other functions in addition to contributing to the structure of lipid bilayers (Delhaize et al. 1999). The best-known role of PS as an enzyme cofactor is as a specific activator of the conventional isoforms of protein kinase C. An important function of PS is also serving as a precursor of PE (Vance and Steenbergen 2005). In the examined wheat seedlings, statistically significant increase was observed only in Se treated shoots.

Analysis of the distribution of the hydrocarbon chains showed that palmitic acid (C16:0) and stearic acid (C18:0) were the most predominant saturated fatty acids in both wheat organs, whereas oleic acid (C18:1), linoleic acid

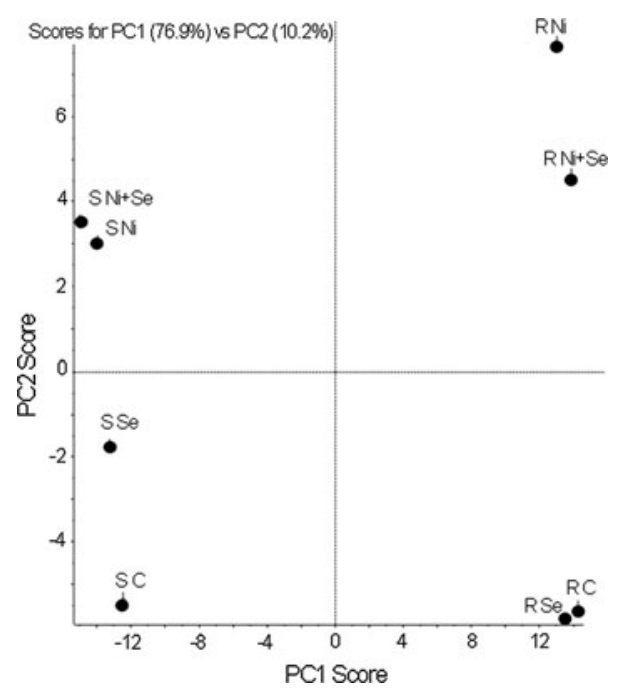

Fig. 5 Score plot from principal component analysis (PCA) of lipidome data from wheat seedlings samples. $(R)$ root, $(S)$ shoot; (C) control, (Ni) nickel, (Se) selenium
(C18:2) and linolenic acid (C18:3) were the major unsaturated species (Figs. 3, 4). This was in accordance with our previous work (Gajewska et al. 2012).

Among the root phospholipids, Ni-treated seedlings exhibited a significant decrease in the content of phospholipid molecular species, especially, those containing C18:3 fatty acid: PA 16:0/18:3, PA 18:2/18:3, PA 18:3/18:3 and a statistically significant increase in PC 18:1/18:2, PE 16:0/ 18:2, PS 16:0/18:2 and PS 18:1/18:2 (Fig. 3). Similar results were obtained for $\mathrm{Ni}+\mathrm{Se}$-treated seedlings. On the other hand, Se influenced only PC 16:0/18:2 and PE 16:0/18:2.

In the shoot phospholipids statistically significant changes were found less frequently than in the roots. A decline was observed for PE 16:0/18:2 and PS 18:1/18:3 in $\mathrm{Ni}$ and/or Se-treated samples. Also, the amount of PC 18:1/ 18:2 and PG 16:0/18:3 decreased in Ni presence (Fig. 4). Among the shoot phospholipids the amount of PS 16:0/ 18:1 increased in seedlings exposed to the examined elements, while the level of PE 18:1/18:2, PG 16:0/18:2 and PA 16:0/18:2, PE 18:2/20:1 elevated in Ni and Se presence, respectively. Results for Ni-treated shoots are consistent with the observations concerning the influence of $\mathrm{Cd}, \mathrm{Pb}$ and $\mathrm{Zn}$ on lipid metabolism in plants (Verdoni et al. 2001; Nouairi et al. 2006). Also, in our previous work we documented that in Ni presence the amount of C18:3 fatty acid extracted from wheat seedlings decreased significantly (Gajewska et al. 2012).

To investigate the metabolic differences in phospholipid species between wheat seedlings exposed to the elements, PCA analysis was used, based on the relative percentages of PLs (Fig. 5). There were 39 variables (species of lipids) to describe abundances of each lipid in each organ extract. Projection of the resulting sample scores for the first and second principal components, which together accounted for $87.1 \%$ of the total sample variance, showed the similarities and differences between the samples. The greatest differences were between roots and shoots, which were separated primarily along the PC1 axis; PC1 explained $76.9 \%$ of the variance of the dataset. Along the PC2 axis, which explained $10.2 \%$ of the variance, were primarily separated control and Ni-treated samples. Results of PCA suggest that were considerable differences between $\mathrm{Ni}$ and control samples, and between roots and shoots. Moreover, the obtained results indicated that the changes of lipidome in samples treated with $\mathrm{Ni}$ and $\mathrm{Ni}+\mathrm{Se}$ were similar. On the other hand, the differences in lipids profile caused by Se used individually were greater in shoots than in roots.

\section{Conclusion}

Our work is the first investigation of the PL fraction of shoots and roots of wheat seedlings treated with $\mathrm{Ni}$ and/or 
Se. Although Se addition to the growth medium resulted in lower uptake of $\mathrm{Ni}$ and improved growth of wheat seedlings, PCA analysis demonstrated that Se did not counteract the effects caused by $\mathrm{Ni}$ on wheat phospholipids. It can be concluded that the changes in the phospholipids profile may be considered as an indicator of $\mathrm{Ni}$ stress.

Acknowledgments This study was supported by the University of Łódź Grants no. 506/819 and 506/802.

Open Access This article is distributed under the terms of the Creative Commons Attribution License which permits any use, distribution, and reproduction in any medium, provided the original author(s) and the source are credited.

\section{References}

Ben Ammar W, Nouairi I, Zarrouk M, Jemal F (2007) Cadmium stress induces changes in the lipid composition and biosynthesis in tomato (Lycopersicon esculentum Mill.) leaves. Plant Growth Regul 53:75-85

Chen C, Huang D, Liu J (2009) Functions and toxicity of nickel in plants: recent advances and future prospects. Clean 37:304-313

Darwish E, Testerink C, Khalil M, El-Shihy O, Munnik T (2009) Phospholipid signaling responses in salt-stressed rice leaves. Plant Cell Physiol 50:986-997

Delhaize E, Hebb DM, Richards KD, Lin JM, Ryan PR, Gardner RC (1999) Cloning and expression of a wheat (Triticum aestivum L.) phosphatidylserine synthase cDNA. Overexpression in plants alters the composition of phospholipids. J Biol Chem 274:7082-7088

Filek M, Keskinen R, Hartikainen H, Szarejko I, Janiak A, Miszalski Z, Golda A (2008) The protective role of selenium in rape seedlings subjected to cadmium stress. J Plant Physiol 165:833-844

Gajewska E, Skłodowska M (2007) Effect of nickel on ROS content and antioxidative enzyme activities in wheat leaves. Biometals 20:27-36

Gajewska E, Skłodowska M (2009) Nickel-induced changes in nitrogen metabolism in wheat shoots. J Plant Physiol 166:1034-1044

Gajewska E, Bernat P, Długoński J, Skłodowska M (2012) Effect of nickel on membrane integrity, lipid peroxidation and fatty acid composition in wheat seedlings. J Agron Crop Sci 198:286-294

Gajewska E, Niewiadomska E, Tokarz K, Słaba M, Skłodowska M (2013) Nickel-induced changes in carbon metabolism. J Plant Physiol 170:369-377

Hasanuzzaman M, Hossain MA, Fujita M (2010) Selenium in higher plants: physiological role, antioxidant metabolism and abiotic stress tolerance. J Plant Sci 5:354-375

Hasanuzzaman M, Hossain MA, Fujita M (2012) Exogenous selenium pretreatment protects rapeseed seedlings from cadmium-induced oxidative stress by upregulating antioxidant defense and methylglyoxal detoxification systems. Biol Trace Elem Res 149:248-261

Hawrylak B, Matraszek R, Szymańska M (2007) Response of lettuce (Lactuca sativa $L$.) to selenium in nutrient solution contaminated with nickel. Veg Crops Res Bull 67:63-70
He PP, Lu XZ, Wang GY (2004) Effects of Se and Zn supplementation on the antagonism against $\mathrm{Pb}$ and $\mathrm{Cd}$ in vegetables. Environ Int 30:167-172

Hummel J, Segu S, Li Y, Irgang S, Jueppner J, Giavalisco P (2011) Ultra performance liquid chromatography and high resolution mass spectrometry for the analysis of plant lipids. Front Plant Sci 2:54

Mansour MMF, Al-Mutawa MM, Salama KHA, Abou Hadid AMF (2002) Effect of $\mathrm{NaCl}$ and polyamines on plasma membrane lipid of wheat roots. Biol Plant 45:235-239

Nouairi I, Ben Ammar W, Ben Youssef N, Ben Miled DD, Ghorbal MH, Zarrouk M (2006) Variations in membrane lipid metabolism in Brassica juncea and Brassica napus leaves as a response to cadmium exposure. J Agron 5:299-307

Ouariti O, Boussama N, Zarrouk M, Cherif A, Ghorbal MH (1997) Cadmium- and copper-induced changes in tomato membrane lipids. Phytochem 45:1343-1350

Pereyra MA, Zalazar CA, Barassi CA (2006) Root phospholipids in Azospirillum-inoculated wheat seedlings exposed to water stress. Plant Physiol Biochem 44:873-879

Quartacci MF, Cosi E, Navari-Izzo F (2001) Lipids and NADPHdependent superoxide production in plasma membrane vesicles from roots of wheat grown under copper deficiency or excess. J Exp Bot 52:77-84

Shanker K, Mishra S, Srivastava S, Srivatava R, Dass S, Prakash S, Srivastava MM (1996) Effect of selenite and selenate on plant uptake of cadmium by maize (Zea mays). Bull Environ Contam Toxicol 56:419-424

Su MK, Bremer DJ, Jeannotte R, Welti R, Yang C (2009) Membrane lipid composition and heat tolerance in cool-season turfgrasses, including a hybrid bluegrass. J Am Soc Hort Sci 134:511-520

Sutoh K, Sanuki N, Sakaki T, Imai R (2010) Specific induction of TaAAPT1, an ER- and Golgi-localized ECPT-type aminoalcoholphosphotransferase, results in preferential accumulation of the phosphatidylethanolamine membrane phospholipid during cold acclimation in wheat. Plant Mol Biol 72:519-531

Testerink C, Munnik T (2005) Phosphatidic acid: a multifunctional stress signaling lipid in plants. Trends Plant Sci 10:368-375

Van Nostrand JD, Arthur JM, Kilpatrick LE, Neely BA, Bertsch PM, Morris PJ (2008) Changes in protein expression in Burkholderia vietnamiensis PR1 301 at $\mathrm{pH} 5$ and 7 with and without nickel. Microbiology 154:3813-3824

Vance JE, Steenbergen R (2005) Metabolism and functions of phosphatidylserine. Prog Lipid Res 44:207-234

Verdoni N, Mench M, Cassagne C, Bessoule JJ (2001) Fatty acid composition of tomato leaves as biomarkers of metal-contaminated soils. Environ Toxicol Chem 20:382-388

Watkinson JH (1966) Fluorometric determination of selenium in biological material with 2,3-diaminonaphthalene. Anal Chem 38:92-97

Welti R, Wang X, Williams TD (2003) Electrospray ionization tandem mass spectrometry scan modes for plant chloroplast lipids. Anal Biochem 314:149-152

Xue T, Hartikainen H, Piironen V (2001) Antioxidative and growthpromoting effect of selenium on senescing lettuce. Plant Soil 237:55-61 